\title{
15th International Symposium on Toxicity Assessment
}

\author{
New Directions in Ecotoxicology and Meeting the Challenges Ahead
}

\author{
Doris W. T. Au
}

Received: 16 May 2012 / Accepted: 21 May 2012 /Published online: 8 June 2012

(C) Springer-Verlag 2012

This special issue of Environmental Science and Pollution Research highlights selected papers presented at the 15th International Symposium on Toxicity Assessment (ISTA15) which was held in Hong Kong, from 3 to 8 July 2011, and officially hosted by the City University of Hong Kong.

In the Asia Pacific region in general, and Hong Kong and China in particular, governments now recognize that proper management of the environment is vital to protect public health and sustain socioeconomic development. In China, rapid population growth and industrial development have occurred in the Pearl River Delta, which is now known as "the world's factory". The emission and disposal of enormous quantities of industrial and domestic waste have exerted unprecedented pressures on our environment. Moreover, many large infrastructural development schemes have caused great damage to our ecosystems and serious economic losses, and have posed significant threats to public health. The impact of these developments needs to be predicted, identified, assessed, monitored, and controlled in order to avoid costly or irreversible mistakes.

ISTA15 brought together academics, professionals, and policy makers to discuss new developments, approaches, and strategies in the fields of toxicity assessment, ecotoxicology, regulatory toxicity, and risk assessment for ensuring environmental safety and public health. A total of 300 registered participants from over 33 countries attended the

Responsible editor: Philippe Garrigues

D. W. T. Au ( $₫)$

Department of Biology \& Chemistry, State Key Laboratory in

Marine Pollution, City University of Hong Kong,

83 Tat Chee Avenue,

Kowloon, Hong Kong, China

e-mail: bhdwtau@cityu.edu.hk
ISTA15 which was characterized by these 10 major scientific themes:

- Biomarkers and bioindicators

- Biotoxins

- Chemical toxicities and mechanisms of action

- Chemicals of global and emerging concerns

- Environmental chemistry and green chemistry

- Environmental safety and public health

- "Omics" and bioinformatics in (eco)toxicology

- Regulatory toxicology

- Remediation and mitigation

- Risk assessment and environmental management

More than 100,000 synthetic chemicals are used in consumer products today. These represent a variety of structurally diverse chemicals including pharmaceuticals and personal care products (PPCPs), brominated flame retardant (PBDEs), plasticizers, and nanomaterials etc. These chemicals enter the environment through wastewater discharge, product consumption, and product deterioration. Many of these synthetic chemicals are of global and emerging concern as they are persistence in the environment, can be readily absorbed by biota, and accumulated in the food web. Some have already been identified as potential endocrine disruptors and nongenotoxic carcinogens. The presence of only trace concentrations of these emerging chemicals in the environment may pose a significant threat to both wildlife and humans. There are urgent needs to develop effective assessment strategies that can be used to evaluate and predict chemical impacts on individuals and populations. A longstanding barrier to progress in biomarkers development for regulatory toxicology has been the inability of most sub-organismal responses (including molecular, biochemical changes) to indicate significant effects at the populations/communities levels. This has impeded the use of biomarkers in regulation, management, and 
monitoring. ISTA15 highlighted the approaches and effort employed to link biomarker responses at lower biological organization levels to Darwinian fitness traits (i.e., survival, growth, reproduction, and recruitment), which enable predicting quantitative changes at the populations/communities levels.

Sixteen papers were accepted and included in this special issue. These papers highlighted current research on unraveling the mode of action and adverse outcomes for a variety of environmental contaminants (including PBDEs, heavy oil, perfluorinated compounds, bisphenol A, phthalate, PPCPs), using a suite of novel in vitro assays and in vivo models. The immune and neuroendocrine systems interact and communicate closely with each other. Immunotoxic and neurotoxic effects of environmental toxicants on fish were highlighted in this special issue. The ultimate goal and challenge ahead is to develop a suite of immunotoxic/neurotoxic endpoints that are useful to delineate the cause and effects, identify adaptive response, and predict adverse biological outcomes with population-level significance. Yet, current findings have recommended future direction for risk assessment of immune-modulatory chemicals must include parallel evaluation of both male and female individuals. In this regard, the small size medaka (the marine Oryzias melastigma and the freshwater Oryzias latipes) offer additional advantage as experimental models for toxicity assessment, because of their distinct gender dimorphism and the presence of sex determining dmy gene in $O$. latipes.

Sophisticated "omics" technologies (transcriptomics, proteomics, and metabolomics) have been proven powerful in generating large amount of mechanistic information. A huge knowledge gap, however, is evident between generation of "omics" data and incorporation of these "omic" data for risk assessment. There is an urgent need for ecotoxicologists and computational scientists to develop easy-tooperate models for effective synthesis of "omics" data to predict population-level effects and support quantitative ecological risk assessment. On the other hand, toxicological studies of mixtures effects remain largely at infancy. For risk assessment for mixtures, a theoretical overall risk probability based method is proposed for the quantification of synergistic and antagonistic effects in health risk assessment. A major breakthrough is certainly needed in this research area.

In keeping with ISTA tradition, three Best Student Awards were presented for (1) an Outstanding Oral Presentation for Progressed Postgraduate Student, (2) an Outstanding Oral Presentation for Early Stage Postgraduate Student, and (3) an Outstanding Poster Presentation for Postgraduate Student. The winners of these awards were, respectively, $\mathrm{Mr}$ Napo K.M. Cheung, Hong Kong (Hypoxia and liver development), Mr T.C.E. Lau, Hong Kong (Thermal tolerance of amphibians and their invasive predator in a polluted environment) and Mr Andy L.Y. Cheung, Hong Kong (Hormetic effect induced by alpha-particle-induced stress communicat$e d$ in vivo between zebrafish embryos).

ISTA symposia have been held in 15 different cities in 12 countries on a biennial basis since 1983. The first two series of ISTA were held in Burlington (1983) and Banff (1985), Canada. Thereafter, the Symposium has traveled the globe: Valencia, Spain (1987), Las Vegas, USA (1989), Kurashiki, Japan (1991), Berlin, Germany (1993), Santiago, Chile (1995), Perth, Australia (1997), Pretoria, South Africa (1999), Quebec City, Canada (2001), Vilnius, Lithuania (2003), Skiathos island, Greece (2005), Toyama, Japan (2007), Metz, France (2009) and Hong Kong, China (2011). The next symposium, ISTA16, will be held in Cape Town, South Africa, in 2013. We look forward to seeing all past ISTA participants as well as new faces at the next meeting.

Acknowledgments The ISTA15 Organizing Committee extends its sincere thanks to Environmental Science and Pollution Research editor-in-chief Prof Philippe Garrigues and to Springer, the journal's publisher, for their full support to SI ISTA15. We are grateful to the ESPR Editorial Board and reviewers for undertaking careful review of manuscripts submitted to this special issue. ISTA15 was sponsored by the PROCORE-France/Hong Kong Joint Research Scheme, The Croucher Foundation and the KC Wong Education Foundation.

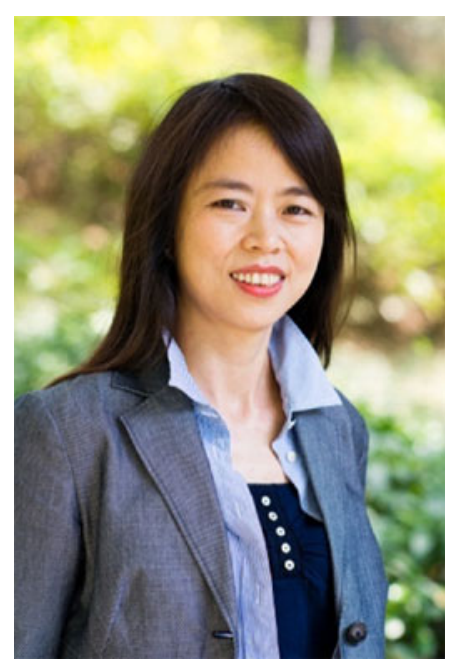

Dr Doris W. T. Au is an associate professor at the Department of Biology and Chemistry, City University of Hong Kong. Her research focuses on molecular toxicology and biomarkers development. The primary objective is to study sub-organismal responses to environmental stresses, aiming to elucidate the underlying mechanisms of action, and develop novel biomarkers for relating to Darwinian fitness traits and assessment of ecological risks. She has published seven book chapters and over 80 papers in leading international journals in biology and environmental sciences. She was awarded the Distinguished Toxicological Lecturer by the University of Saskatchewan, Canada in 2008. Dr. Au has been the core Consultant of many milestone projects commissioned by the Hong Kong government (with a total contract value exceeded \$2 million), including the development of bioindicator systems for marine pollution monitoring, development of chronic tests for toxicity identification and impact assessment, and establishment of marine water quality objectives for Hong Kong. In the past years, she has organized and conducted nine regional training courses for various international organizations (including United Nations and Asia Pacific Economic Cooperation) and the State Key Laboratory of Marine Environmental Sciences and the State Key Laboratory in Marine Pollution, China. 